\title{
Clinical ANATOMY OF THE LUMBAR SPINE AND SACRUM
}

\section{Third Edition:}

By: $\quad$ Nikolai Bogduk

Publisher: Churchill Livingstone, 1997.

This is the third edition of an excellent source relating to the anatomical, biochemical and pathological issues related to the lumbar spine and sacrum. It is well illustrated and presented, making it easy to access relevant information. It is well referenced and states scientific findings clearly. It brings us a collection and an analysis of the latest scientific research regarding the clinical causes of low back pain which is most enlightening.
Chapter 1 through to 12 deals extensively with the anatomical, biochemical and biomechanical considerations of the lumbar spine and sacrum. The effects of age in the lumbar spine is dealt with in an informative way. The chapter dealing with the possible sources and causes of low back pain is an excellent overview of pertinent literature. He deals with the anatomical areas that may cause back pain which is essential to an understanding of the pathological processes at work. It is through this understanding that logical treatment can be offered. He makes the observation that the most popularly held clinical conditions which are postulated to cause back pain such as trigger points, ligament and muscle pain are associated with the smallest amount of scientific evidence. He discusses the more well researched, and often less popular hypothesis in detail. New insights into these issues are explored in a most accessible way. This is an essential book for all physiotherapists involved in the treatment and long term rehabilitation of people with pain related to lumbar spine and/or sacrum.

Reviewed by: Trish Wallner - Schlotfeldt 